Supporting Information

\title{
Ultrasensitive Plasmon-Free Surface-Enhanced Raman Spectroscopy with Femtomolar Detection Limit from 2D van der Waals
}

\section{Heterostructure}

Jihyung Seo,,$^{\dagger}$ Junghyun Lee, ${ }^{\dagger}$ Yongchul Kim,,$\$$ Donghwan Koo, ${ }^{\dagger}$ Geunsik Lee, ${ }^{\S}$ and Hyesung $\operatorname{Park}^{\dagger, *}$

†Department of Energy Engineering, School of Energy and Chemical Engineering, Low Dimensional Carbon Materials Center, Perovtronics Research Center, Ulsan National Institute of Science and Technology (UNIST), Ulsan 44919, Republic of Korea

${ }^{\S}$ Department of Chemistry, Ulsan National Institute of Science and Technology (UNIST), Ulsan 44919, Republic of Korea

${ }^{*}$ Corresponding author

E-mail address: hspark@unist.ac.kr 


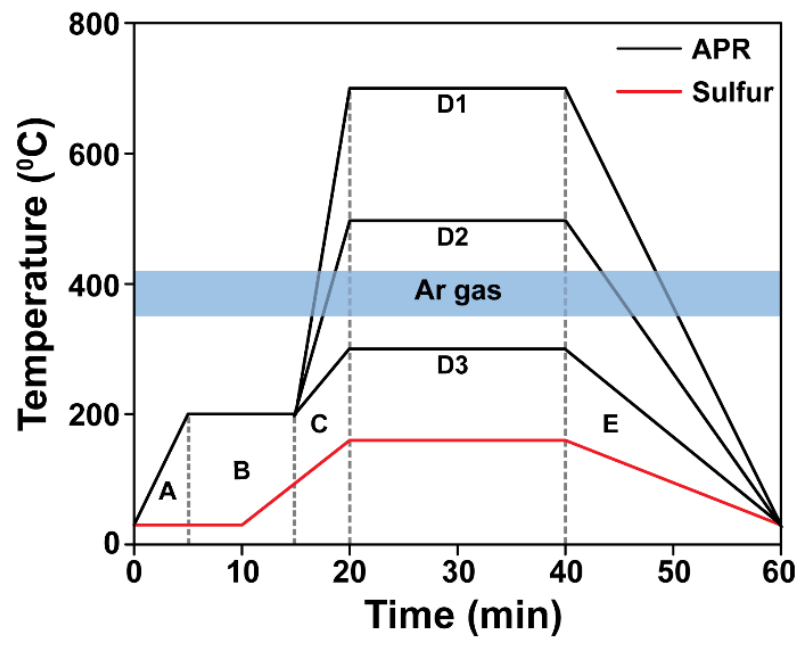

Figure S1. Temperature profiles and gas composition in the growth of $\mathrm{ReO}_{\mathrm{x}} \mathrm{S}_{\mathrm{y}}$ thin film via solution phase deposition (A: ramping process, B: annealing process, C: ramping process, D: growth process, E: cooling process).

\begin{tabular}{c|ccc}
\hline Process & Stage & Time (min) & Temperature $\left({ }^{\circ} \mathbf{C}\right)$ \\
\hline Ramping & A & 5 & RT $\rightarrow 200$ \\
\hline Annealing & B & 10 & 200 \\
\hline Ramping & C & 5 & $200 \rightarrow$ GT \\
\hline \multirow{2}{*}{ Growth } & D1 & 20 & 700 \\
& D2 & 20 & 500 \\
\hline Cooling & D3 & 20 & 300 \\
\hline & E & 20 & GT $\rightarrow$ RT \\
\hline
\end{tabular}

Table S1. Summary of the growth stage for the synthesis of $\mathrm{ReO}_{\mathrm{x}} \mathrm{S}_{\mathrm{y}}-\mathrm{LT} / \mathrm{MT} / \mathrm{HT}$ from Figure S1. 

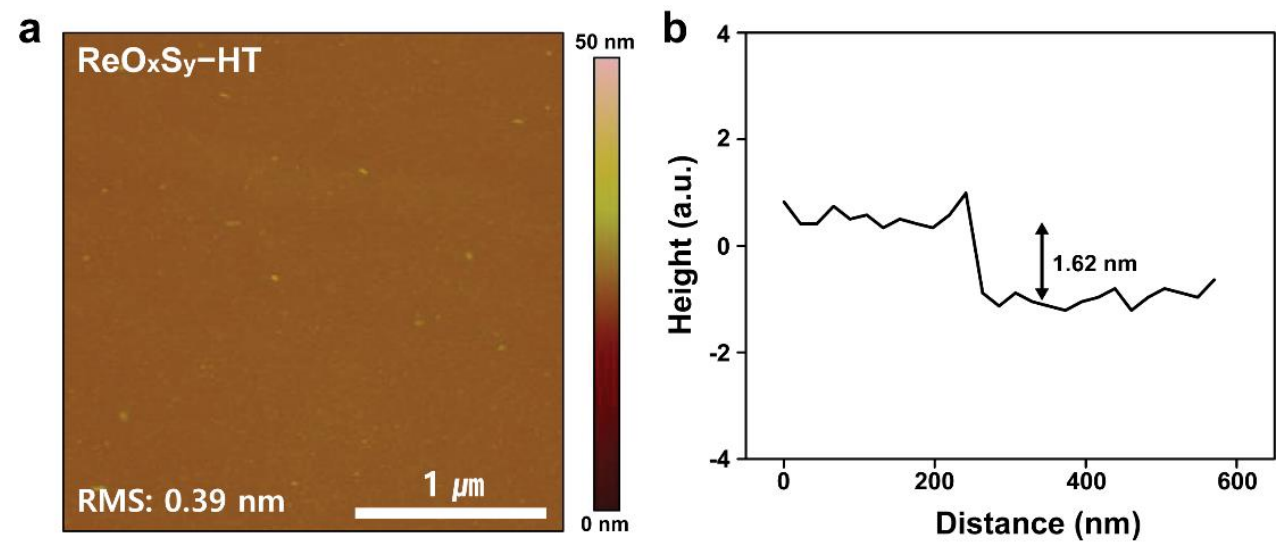

Figure S2. AFM image and thickness of $\mathrm{ReO}_{\mathrm{x}} \mathrm{S}_{\mathrm{y}}-\mathrm{HT}$ synthesized on sapphire substrate via solution phase deposition.
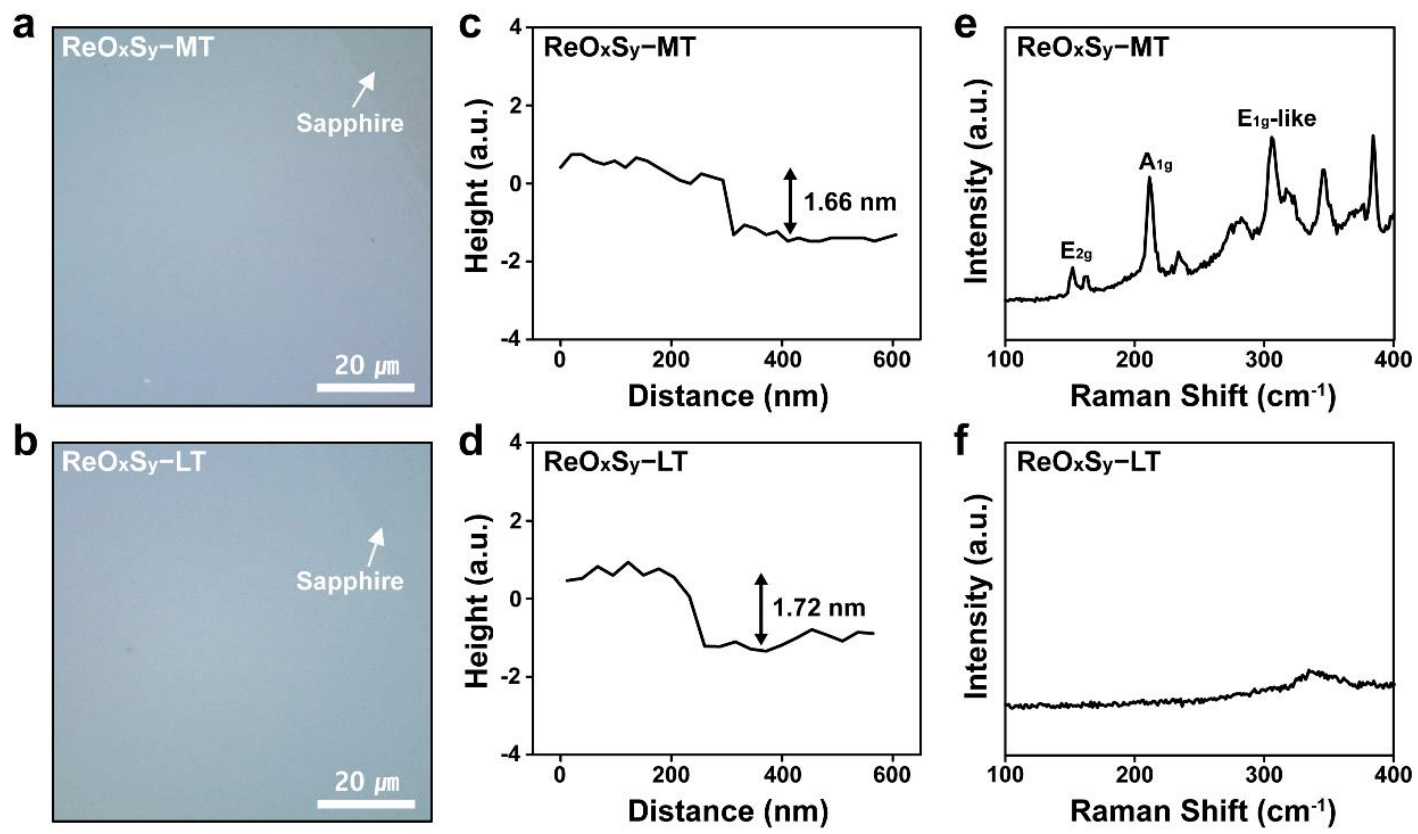

Figure S3. (a,b) OM images, (c,d) film thickness, and (e,f) Raman spectra for the assynthesized $\mathrm{ReO}_{\mathrm{x}} \mathrm{S}_{\mathrm{y}}-\mathrm{LT} / \mathrm{MT}$. 


\begin{tabular}{c|ccc}
\hline $\operatorname{ReO}_{\mathbf{x}} \mathbf{S}_{\mathbf{y}}$ & $\boldsymbol{R e}(\boldsymbol{\%})$ & $\mathbf{O}(\mathbf{\%})$ & $\mathbf{S}(\mathbf{\%})$ \\
\hline LT & 33.13 & 47.65 & 19.22 \\
\hline MT & 32.84 & 19.54 & 47.62 \\
\hline HT & 33.68 & 7.04 & 59.28 \\
\hline
\end{tabular}

Table S2. Atomic percentages of Re, $\mathrm{O}$, and $\mathrm{S}$ atoms in the as-synthesized $\mathrm{ReO}_{\mathrm{x}} \mathrm{S}_{\mathrm{y}}-\mathrm{LT} / \mathrm{MT} / \mathrm{HT}$. As the sulfurization temperature increases from LT to HT, oxygen concentrations in the $\mathrm{ReO}_{\mathrm{x}} \mathrm{S}_{\mathrm{y}}$ lattice changes from 47.65 to $7.04 \%$.

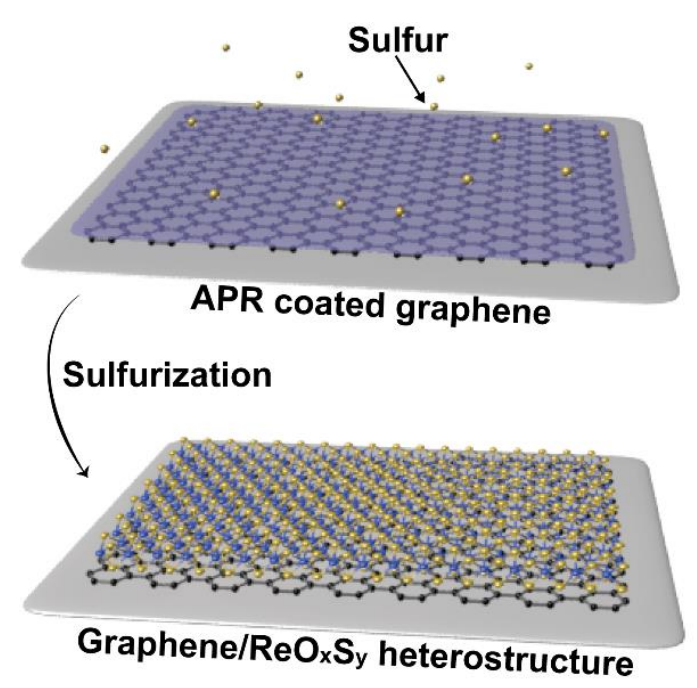

Figure S4. Synthesis process for the graphene/ $\mathrm{ReO}_{\mathrm{x}} \mathrm{S}_{\mathrm{y}}$ vertical heterostructure through solution phase deposition. 


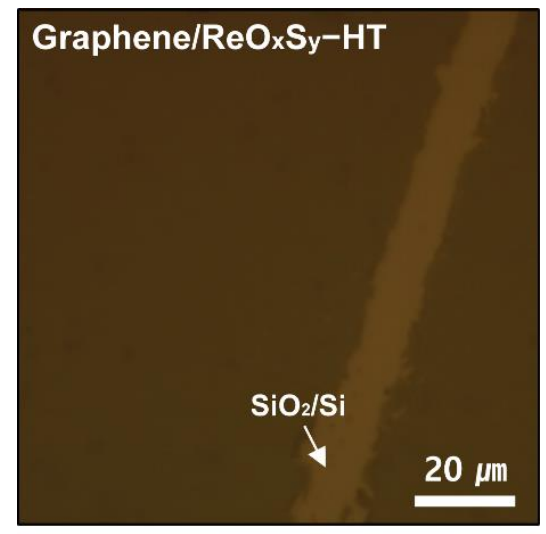

Figure S5. OM image of the graphene/ $\mathrm{ReO}_{\mathrm{x}} \mathrm{S}_{\mathrm{y}}-\mathrm{HT}$ vertical heterostructure synthesized on $\mathrm{SiO}_{2} / \mathrm{Si}$ substrate by using solution precursors.

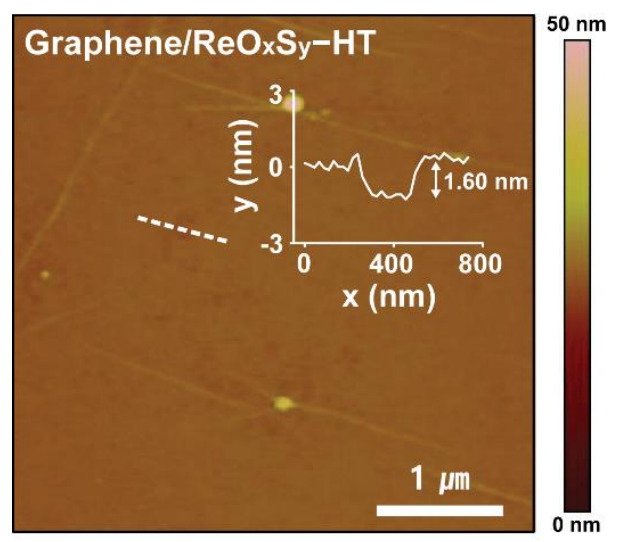

Figure S6. AFM image of the graphene/ $\mathrm{ReO}_{\mathrm{x}} \mathrm{S}_{\mathrm{y}}-\mathrm{HT}$ heterostructure. The inset shows the height profile of the $\mathrm{ReO}_{\mathrm{x}} \mathrm{S}_{\mathrm{y}}$ film on graphene from the dewetted region marked with dotted line. 

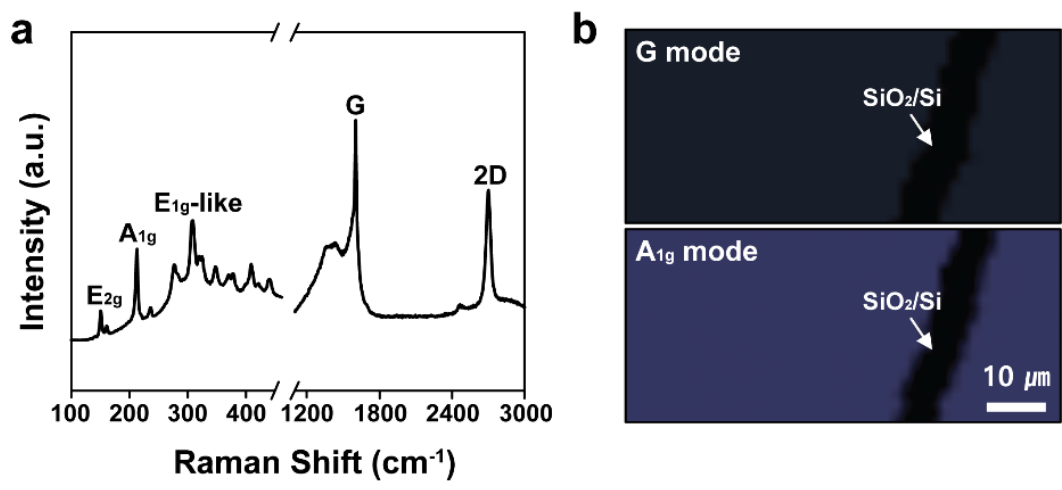

Figure S7. (a) Raman spectrum of the vibrational modes of $\mathrm{ReO}_{x} \mathrm{~S}_{\mathrm{y}}$ and graphene in graphene/ $\mathrm{ReO}_{\mathrm{x}} \mathrm{S}_{\mathrm{y}}-\mathrm{HT}$ vertical heterostructure. (b) Raman mapping of G mode for graphene and $\mathrm{A}_{1 \mathrm{~g}}$ mode for $\mathrm{ReO}_{\mathrm{x}} \mathrm{S}_{\mathrm{y}}$ from the graphene/ $\mathrm{ReO}_{\mathrm{x}} \mathrm{S}_{\mathrm{y}}-\mathrm{HT}$ heterostructure.
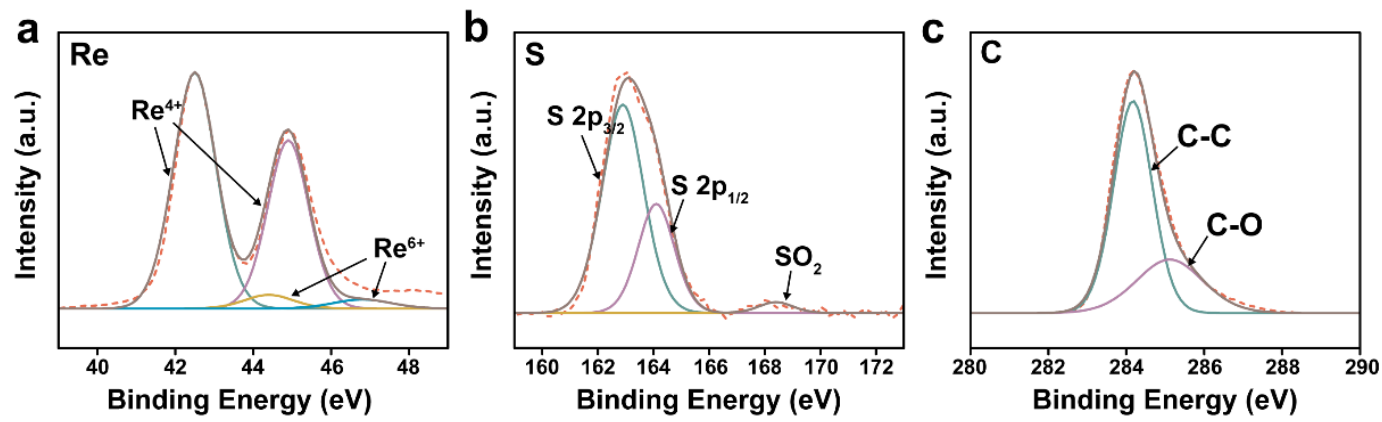

Figure S8. XPS spectra of the (a) Re 4f, (b) S 2p, and (c) C 1s peaks from the graphene/ $\mathrm{ReO}_{\mathrm{x}} \mathrm{S}_{\mathrm{y}}-\mathrm{HT}$ heterostructure film. 


\begin{tabular}{c|c}
\hline Frequency $\left(\mathbf{c m}^{-1}\right)$ & Assignment \\
\hline 614 & C-C-C ring in-plane bend \\
\hline 776 & C-H out-of-plane bend \\
\hline 1131 & C-H in-plane bend \\
\hline 1185 & C-C stretching vibration bend \\
\hline 1312 & Aromatic C-C stretching \\
\hline 1363 & Aromatic C-C stretching \\
\hline 1419 & Aromatic C-C stretching / C-H vibration \\
\hline 1506 & Aromatic C-C stretching \\
\hline 1532 & Aromatic C-C stretching \\
\hline 1575 & Aromatic C-C stretching \\
\hline 1601 & Aromatic C-C stretching / C-H vibration \\
\hline 1650 & Aromatic C-C stretching \\
\hline
\end{tabular}

Table S3. The Raman bands and assignments for R6G molecule. ${ }^{1,2}$
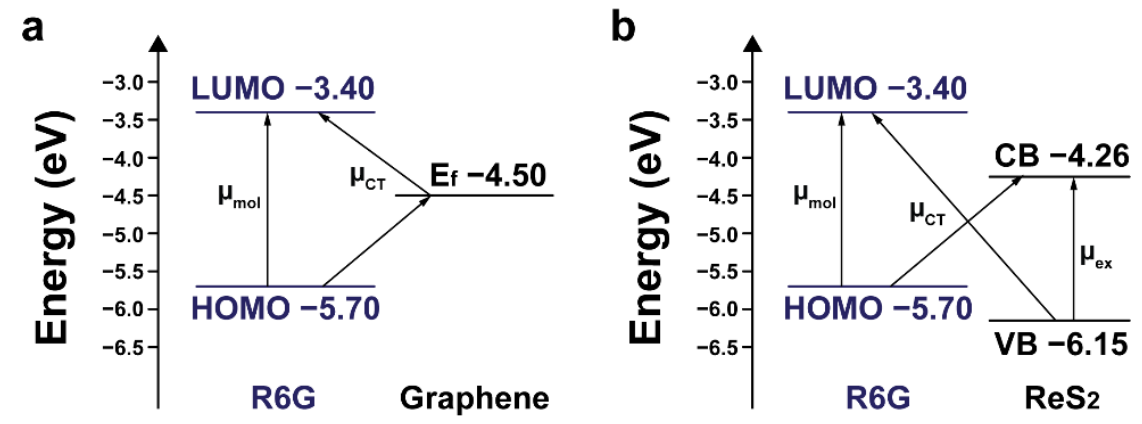

Figure S9. Energy level diagram and charge transfer resonance in (a) R6G-graphene and (b) R6G-ReS 2 system. 

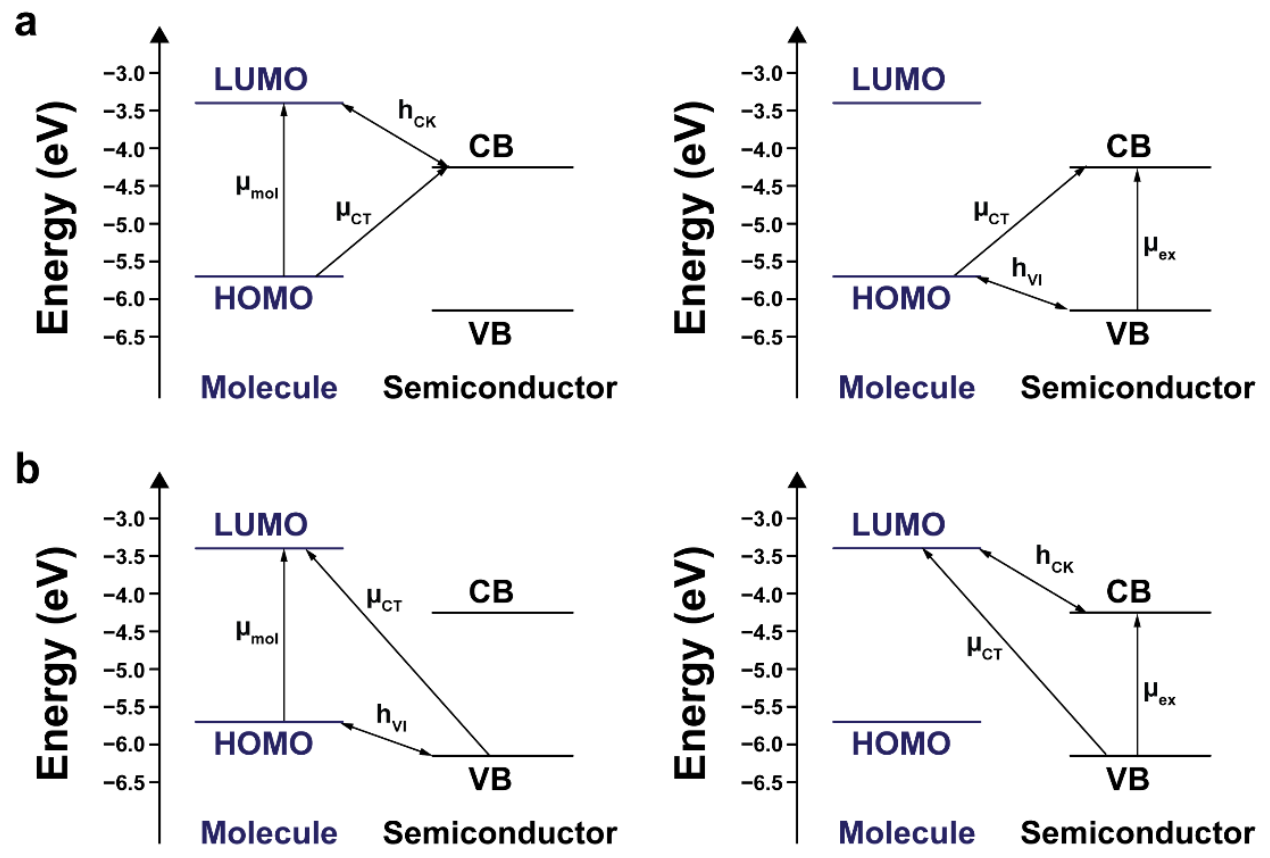

Figure S10. Charge transfer resonance (a) from molecule to semiconductor and (b) from semiconductor to molecule, which is coupled with molecular and exciton resonances through the Herzberg-Teller constants in molecule-semiconductor system. 

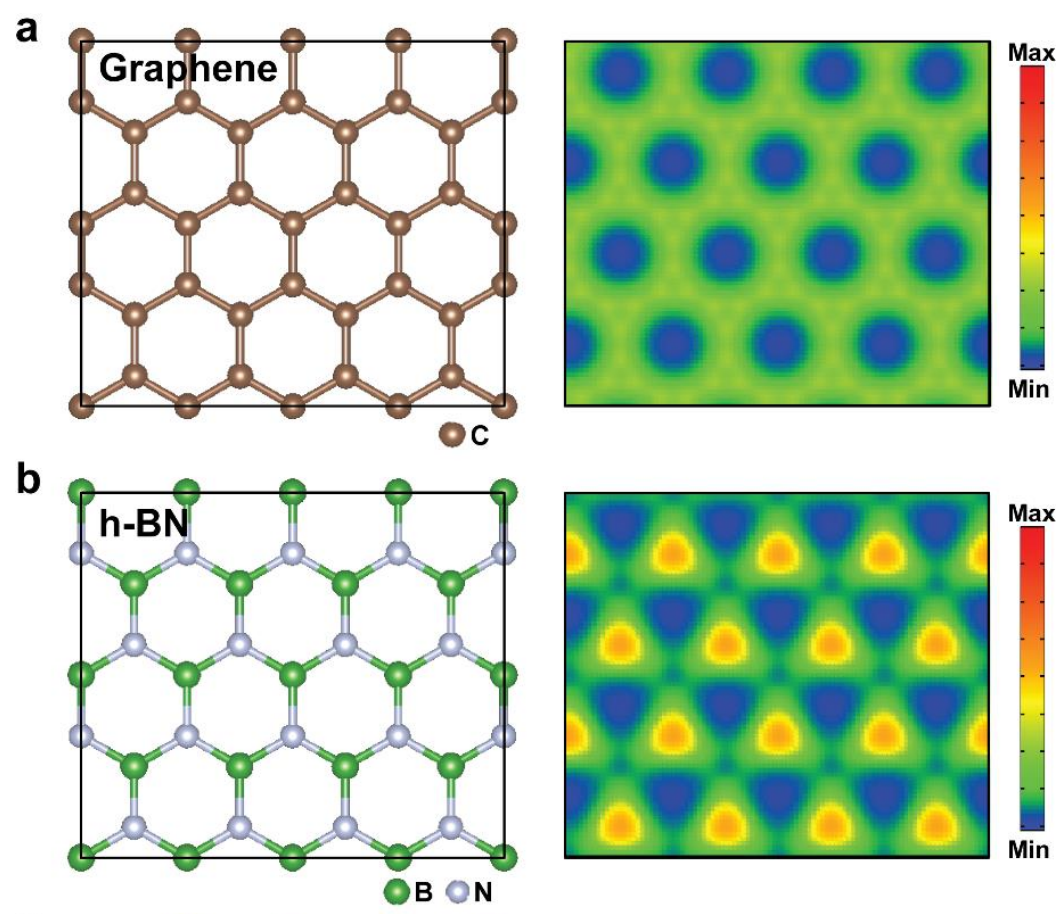

C

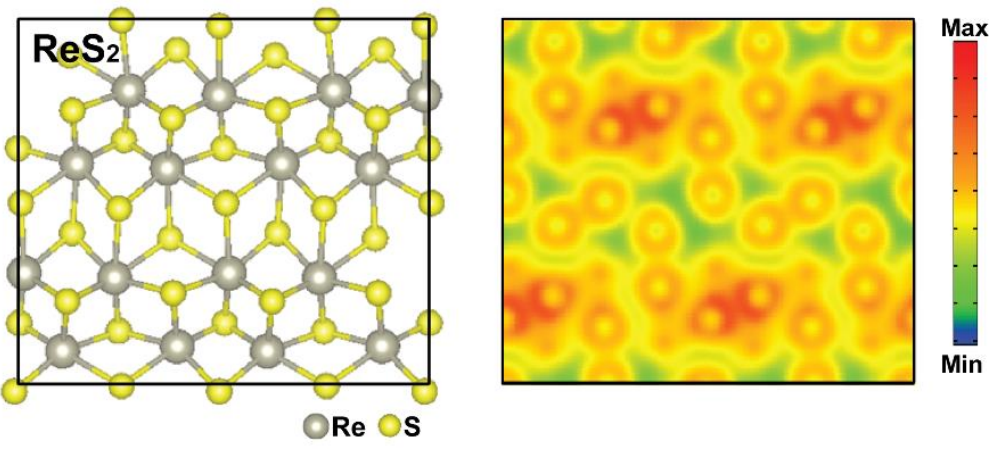

Figure S11. Atomic structure and charge density plot of (a) graphene, (b) h-BN, and (c) $\mathrm{ReS}_{2}$. 


\begin{tabular}{|c|c|c|c|c|c|}
\hline Sample & Synthesis method & $\begin{array}{c}\text { Probe } \\
\text { molecule }\end{array}$ & LOD & $\begin{array}{c}\text { Excitation } \\
\text { wavelength }\end{array}$ & Reference \\
\hline Graphene & $\begin{array}{l}\text { Mechanical } \\
\text { exfoliation }\end{array}$ & R6G & $1 \times 10^{-5} \mathrm{M}$ & $514 \mathrm{~nm}$ & 3 \\
\hline Graphene & $\begin{array}{l}\text { Mechanical } \\
\text { exfoliation }\end{array}$ & $\mathrm{CuPc}$ & $1 \times 10^{-6} \mathrm{M}$ & $633 \mathrm{~nm}$ & 4 \\
\hline N-doped graphene & CVD & R6G & $1 \times 10^{-8} \mathrm{M}$ & $514 \mathrm{~nm}$ & 5 \\
\hline $\begin{array}{l}\text { Mildly reduced } \\
\text { GO }\end{array}$ & $\begin{array}{c}\text { Modified } \\
\text { Hummers' method }\end{array}$ & $\mathrm{RhB}$ & $5 \times 10^{-8} \mathrm{M}$ & $514 \mathrm{~nm}$ & 6 \\
\hline $\begin{array}{c}\text { Plasma modified } \\
\mathrm{MoS}_{2}\end{array}$ & $\begin{array}{l}\text { Mechanical } \\
\text { exfoliation }\end{array}$ & R6G & $1 \times 10^{-6} \mathrm{M}$ & $532 \mathrm{~nm}$ & 7 \\
\hline $\begin{array}{c}1 \mathrm{~T}-\mathrm{MoSe}_{2} \\
\text { (n-butyl lithium) }\end{array}$ & $\begin{array}{l}\text { Chemical } \\
\text { exfoliation }\end{array}$ & R6G & $1 \times 10^{-8} \mathrm{M}$ & $532 \mathrm{~nm}$ & 8 \\
\hline $1 \mathrm{~T}-\mathrm{MoS}_{2}(\mathrm{NaK})$ & $\begin{array}{l}\text { Chemical } \\
\text { exfoliation }\end{array}$ & $\mathrm{CV}$ & $1 \times 10^{-8} \mathrm{M}$ & $532 \mathrm{~nm}$ & 9 \\
\hline $\mathrm{ReS}_{2}$ & CVD & R6G, MB & $1 \times 10^{-9} \mathrm{M}$ & $532,633 \mathrm{~nm}$ & 10 \\
\hline $\begin{array}{c}\text { Oxygen- } \\
\text { substituted } \mathrm{MoS}_{2} \\
\end{array}$ & $\begin{array}{c}\text { Hydrothermal } \\
\text { synthesis }\end{array}$ & R6G & $1 \times 10^{-7} \mathrm{M}$ & $532 \mathrm{~nm}$ & 11 \\
\hline $1 \mathrm{~T}^{\prime}-\mathrm{W}(\mathrm{Mo}) \mathrm{Te}_{2}$ & CVD & R6G & $4(40) \times 10^{-14} \mathrm{M}$ & $532 \mathrm{~nm}$ & 12 \\
\hline $\mathrm{MoS}_{2} \mathrm{QD} / \mathrm{rGO}$ & $\begin{array}{c}\text { Solvothermal } \\
\text { method/Modified } \\
\text { Hummers' method }\end{array}$ & R6G & $1 \times 10^{-9} \mathrm{M}$ & $532 \mathrm{~nm}$ & 13 \\
\hline Graphene/ReO $\mathrm{O}_{\mathbf{y}}$ & CVD & R6G & $1 \times 10^{-15} \mathrm{M}$ & $532 \mathrm{~nm}$ & This work \\
\hline
\end{tabular}

Table S4. Summary of the SERS performance based on 2D materials in the literature.

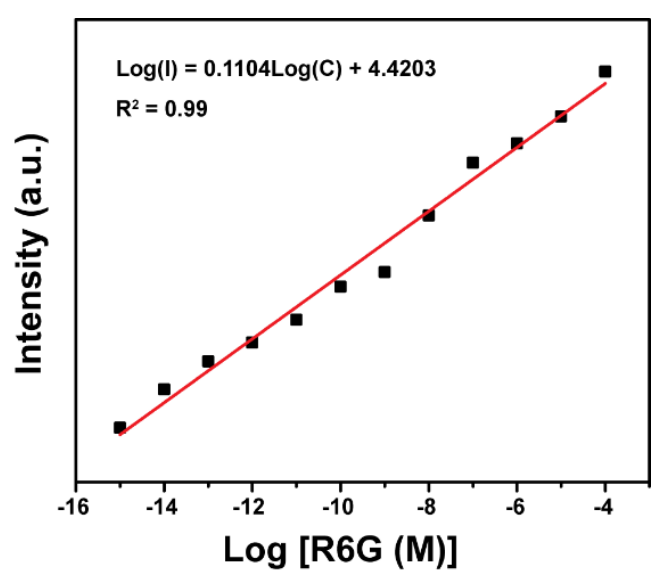


Figure S12. The SERS intensity of R6G on the graphene/ $\mathrm{ReO}_{x} \mathrm{~S}_{y}-\mathrm{MT}$ at $614 \mathrm{~cm}^{-1}$ as function of R6G concentration.

\section{REFERENCES}

(1) Stockburger, M.; Hildebrandt, P. J. Phys. Chem. 1984, 88, 5935-5944.

(2) Liao, F.; Cheng, L.; Li, J.; Shao, M.; Wang, Z.; Lee, S.-T. J. Mater. Chem. C 2013, 1, $1628-1632$.

(3) Xie, L.; Ling, X.; Fang, Y.; Zhang, J.; Liu, Z. J. Am. Chem. Soc. 2009, 131, 9890-9891.

(4) Xu, H.; Xie, L.; Zhang, H.; Zhang, J. ACS Nano 2011, 5, 5338-5344.

(5) Feng, S.; dos Santos, M. C.; Carvalho, B. R.; Lv, R.; Li, Q.; Fujisawa, K.; Elias, A. L.; Lei, Y.; Perea-Lopez, N.; Endo, M.; Pan, M.; Pimenta, M. A.; Terrones, M. Sci. Adv. 2016, 2, e1600322.

(6) Yu, X.; Cai, H.; Zhang, W.; Li, X.; Pan, N.; Luo, Y.; Wang, X.; Hou, J. G. ACS Nano 2011, $5,952-958$.

(7) Sun, L.; Hu, H.; Zhan, D.; Yan, J.; Liu, L.; Teguh, J. S.; Yeow, E. K. L.; Lee, P. S.; Shen, Z. Small 2014, 10, 1090-1095.

(8) Yin, Y.; Miao, P.; Zhang, Y.; Han, J.; Zhang, X.; Gong, Y.; Gu, L.; Xu, C.; Yao, T.; Xu, P.; Wang, Y.; Song, B.; Jin, S. Adv. Funct. Mater. 2017, 27, 1606694.

(9) Er, E.; Hou, H.-L.; Criado, A.; Langer, J.; Moller, M.; Erk, N.; Liz-Marzan, L. M.; Prato, M. Chem. Mater. 2019, 31, 5725-5734.

(10) Miao, P.; Qin, J.-K.; Shen, Y.; Su, H.; Dai, J.; Song, B.; Du, Y.; Sun, M.; Zhang, W.; Wang, H.-L.; Xu, C.-Y.; Xu, P. Small, 2018, 14, 1704079.

(11) Zheng, Z.; Cong, S.; Gong, W.; Xuan, J.; Li, G.; Lu, W.; Geng, F.; Zhao, Z. Nat. Commun. 2017, 8, 1993.

(12) Tao, L.; Chen, K.; Chen, Z.; Cong, C.; Qiu, C.; Chen, J.; Wang, X.; Chen, H.; Yu, T.; Xie, W.; Deng, S.; Xu, J.-B. J. Am. Chem. Soc. 2018, 140, 8696-8704.

(13) Wu, D.; Chen, J.; Ruan, Y.; Sun, K.; Zhang, K.; Xie, W.; Xie, F.; Zhao, X.; Wang, X. J. Mater. Chem. C 2018, 6, 12547-12554. 